\title{
Coronal rain in magnetic bipolar weak fields ${ }^{\star}$
}

\author{
C. Xia, R. Keppens, and X. Fang
}

\begin{abstract}
Centre for mathematical Plasma Astrophysics, Department of Mathematics, KU Leuven, Celestijnenlaan 200B, 3001 Leuven, Belgium

e-mail: chun.xia@kuleuven.be
\end{abstract}

Received 20 February 2017 / Accepted 25 May 2017

\begin{abstract}
Aims. We intend to investigate the underlying physics for the coronal rain phenomenon in a representative bipolar magnetic field, including the formation and the dynamics of coronal rain blobs.

Methods. With the MPI-AMRVAC code, we performed three dimensional radiative magnetohydrodynamic (MHD) simulation with strong heating localized on footpoints of magnetic loops after a relaxation to quiet solar atmosphere.

Results. Progressive cooling and in-situ condensation starts at the loop top due to radiative thermal instability. The first large-scale condensation on the loop top suffers Rayleigh-Taylor instability and becomes fragmented into smaller blobs. The blobs fall vertically dragging magnetic loops until they reach low- $\beta$ regions and start to fall along the loops from loop top to loop footpoints. A statistic study of the coronal rain blobs finds that small blobs with masses of less than $10^{10} \mathrm{~g}$ dominate the population. When blobs fall to lower regions along the magnetic loops, they are stretched and develop a non-uniform velocity pattern with an anti-parallel shearing pattern seen to develop along the central axis of the blobs. Synthetic images of simulated coronal rain with Solar Dynamics Observatory Atmospheric Imaging Assembly well resemble real observations presenting dark falling clumps in hot channels and bright rain blobs in a cool channel. We also find density inhomogeneities during a coronal rain "shower", which reflects the observed multi-stranded nature of coronal rain.
\end{abstract}

Key words. Sun: corona - Sun: activity - Sun: filaments, prominences - magnetohydrodynamics (MHD) - instabilities

\section{Introduction}

Coronal rain refers to cool and dense elongated plasma blobs or thread segments, which suddenly appear in the low corona, falling along coronal loops all the way down to the solar surface. This phenomenon was first recorded and classified as coronal sunspot prominences, commonly originating in coronal space and pouring down to spot regions (Pettit 1943), and was later observed and clarified as "coronal rain" by its characteristic feature of rapid brightening when approaching the chromosphere in the early 1970s (Kawaguchi 1970). It has been well observed in spectral lines sampling temperature ranges from transition region (TR) to chromosphere (de Groof et al. 2005; Kamio et al. 2011; Antolin et al. 2010; Antolin \& Verwichte 2011). High-resolution spectroscopic observations (Antolin \& Rouppe van der Voort 2012) found that coronal rain is composed of elongated blobs with average widths of $310 \mathrm{~km}$, lengths of $710 \mathrm{~km}$, average temperatures below $7000 \mathrm{~K}$, average falling speed of $70 \mathrm{~km} \mathrm{~s}^{-1}$, and accelerations much smaller than the gravitational acceleration projected along loops. In non-flaring coronal loops, coronal rain is observed to appear during progressive cooling with high-speed downflow and dramatic evacuation in the loops (Schrijver 2001; de Groof et al. 2005). O'Shea et al. (2007) found spectroscopic evidence of plasma condensations taking place in coronal loops and forming coronal rain. Antolin \& Rouppe van der Voort (2012) found that coronal rain often occurs simultaneously in neighboring magnetic strands,

\footnotetext{
* Movies associated to Figs. 3 and 7 are available at http://www . aanda.org
}

forming groups of condensations which are seen as large clumps if they are close enough in proximity. These are called "showers" and can have widths up to a few Mm. Antolin et al. (2015) found that coronal rain is a highly multi-thermal phenomenon with the multi-wavelength emission located very closely. The fragmentary and clumpy appearance of coronal rain blobs at coronal heights becomes more continuous and persistent at chromospheric heights immediately before impact. Rain clumps appear organized in parallel strands with density inhomogeneities in both chromospheric and TR temperatures. The electron density of rain clumps was found to be about $1.8-7.1 \times 10^{10} \mathrm{~cm}^{-3}$, through estimation based on absorption in multiple extreme ultraviolet (EUV) channels. Besides this quiescent coronal rain, which occurs in nonflaring coronal loops with relatively weak variation of energy and mass, flare-driven coronal rain, which appears in postflare loops as a result of catastrophic cooling, often emerges as a bunch of parallel strands extending from loop top to footpoint (Scullion et al. 2014, 2016).

With the phenomena of progressive cooling and in situ condensation, coronal rain is believed to be a representative of the general phenomenon of radiative thermal instability (Parker 1953; Field 1965) in an astrophysical plasma, that occurs whenever energy losses due to radiation overcome the heating input and the resulting cooling further amplifies energy losses. Numerical simulations firstly performed in one-dimensional setups (Antiochos et al. 1999; Karpen et al. 2001; Müller et al. 2003, 2004, 2005; Xia et al. 2011) have demonstrated that a heating input of a coronal loop concentrated at both footpoints, in chromosphere and near TR, can cause the loop to experience thermal non-equilibrium and thermal instability. This leads 
to coronal rain formation or prominence formation depending on the magnetic configuration of the loop. Footpoint heating of coronal loops is supported by observational evidence (Aschwanden 2001) and by hydrostatic coronal loop models (Aschwanden et al. 2001). Three-dimensional (3D) magnetohydrodynamics (MHD) numerical simulations (Hansteen et al. 2010) found a concentration of Joule heating toward the upper chromosphere, TR, and lower corona as a result of the braiding of the magnetic field decreasing exponentially with height. Observations (De Pontieu et al. 2011) have shown a ubiquitous coronal mass supply where chromospheric plasma is accelerated and heated in type II spicules upward into the corona, and then leads to fading of type II spicules in chromospheric spectral line images (De Pontieu et al. 2007; Rouppe van der Voort et al. 2009).

Inspired by the first two-dimensional (2D) evaporationcondensation model demonstrating prominence formation in a magnetic arcade (Xia et al. 2012), Fang et al. (2013) presented the first $2 \mathrm{D}$ coronal rain simulation, in which a large zigzag shape condensation forms across the top regions of a magnetic arcade covering a range of several $\mathrm{Mm}$, then splits into many small blobs with elongated side wings and V-shaped features, descending along both sides of the arcade field lines. They performed statistic analyses to quantify blob widths and lengths, which average $400 \mathrm{~km}$ and $800 \mathrm{~km}$, respectively, and velocities up to $65 \mathrm{~km} \mathrm{~s}^{-1}$, which is smaller than the observed average speed. Later, Fang et al. (2015) extended their model with an increased resolution of $20 \mathrm{~km}$ per cell and much longer time coverage, and studied the blob condensation in detail and found recurrent coronal rain showers to occur in limit cycles. They quantified the thermal structure of blob-corona TR and the variations of density, kinetic energy, and temperature during the impact of rain blobs on the chromosphere. They also showed how high-speed anti-parallel shear flows at two sides of condensations are induced that further facilitate fragmentation of the condensations. Moschou et al. (2015) performed 3D simulations on coronal condensation in a magnetic configuration of a quadrupolar arcade system. In their models, coronal condensations occur in nearly horizontal coronal loop parts, where dense blobs first descend through (weak) local magnetic field undergoing Rayleigh-Taylor instability (RTI). Later on, small blobs start to follow more closely the magnetic field lines in the lower regions near the footpoints and slide down to the chromosphere, behaving like coronal rain. Their magnetic configuration did not represent a typical solar active region where coronal rain preferentially occurs. In a magnetic structure built from an observed magnetogram, Mok et al. (2016) simulated an active region validated with simulated EUV emissions. They found thermal nonequilibrium in a coronal loop leading to cooling and coronal condensation, although their spatial resolution was not enough to obtain coronal rain blobs.

In order to better understand coronal rain in realistic coronal loops, we perform a 3D MHD simulation on quiescent coronal rain in a bipolar magnetic field, which is an idealized magnetic structure of a two-sunspot solar active region. The numerical methods and the simulation strategy are explained in Sect. 2. We present the results in Sect. 3 and end with a conclusion and discussion in Sect. 4.

\section{Numerical method}

We setup a 3D Cartesian simulation box with extensions in $x$ from $-20 \mathrm{Mm}$ to $20 \mathrm{Mm}$, in $y$ from $-30 \mathrm{Mm}$ to $30 \mathrm{Mm}$, and in $z$ from $0 \mathrm{Mm}$ to $60 \mathrm{Mm}$. The solar gravity points along the negative $z$ direction and the $z=0$ bottom surface represents the solar surface. With five levels of adaptive mesh refinement, the mesh has an effective resolution of $384 \times 576 \times 768$, with smallest grid cell size of $78 \mathrm{~km}$. To mimic a magnetic configuration of a simple two-sunspot active region, we use a potential magnetic field composed of two dipoles (Török \& Kliem 2003) with vertical and oppositely directed moments located below the solar surface at $\left(0, y_{\mathrm{c}},-z_{\mathrm{c}}\right)$ and $\left(0,-y_{\mathrm{c}},-z_{\mathrm{c}}\right)$, respectively:

$$
\begin{aligned}
& B_{x}=B_{x+}+B_{x-}, \quad B_{y}=B_{y+}+B_{y-}, \quad B_{z}=B_{z+}+B_{z-}, \\
& B_{x \pm}=3 x\left(x+z_{\mathrm{c}}\right) f_{ \pm}, \\
& B_{y \pm}=3\left(y \mp y_{\mathrm{c}}\right)\left(z+z_{\mathrm{c}}\right) f_{ \pm}, \\
& B_{z \pm}=\left[2\left(z+z_{\mathrm{c}}\right)^{2}-x^{2}-\left(y \mp y_{\mathrm{c}}\right)^{2}\right] f_{ \pm}, \\
& f_{ \pm}=\frac{ \pm B_{\mathrm{c}}}{\left[x^{2}+\left(y \mp y_{\mathrm{c}}\right)^{2}+\left(z+z_{\mathrm{c}}\right)^{2}\right]^{2.5}}
\end{aligned}
$$

where $y_{\mathrm{c}}=10 \mathrm{Mm}, z_{\mathrm{c}}=15 \mathrm{Mm}$, and $B_{\mathrm{c}}=200 \mathrm{G}$. This magnetic setup gives maximal $120 \mathrm{G}$ on the bottom surface $(z=0)$ and about $3 \mathrm{G}$ at a height of $30 \mathrm{Mm}$. Although the magnetic field strength is much weaker than a typical solar active region, these weak magnetic loops may be found in a decayed active region. To construct a solar atmosphere, we let the model obtain a realistic thermal and gravitational stratification by relaxing an initial state, which has a vertical distribution of temperature of about $8000 \mathrm{~K}$ below the TR at a height of $2 \mathrm{Mm}$ with smooth connection (TR at $160000 \mathrm{~K}$ using hyperbolic tangent function) to the upper region where the temperature increases with height in such a way that the vertical thermal conduction flux is always $2 \times 10^{5} \mathrm{erg} \mathrm{cm}^{-2} \mathrm{~s}^{-1}$. The density is then calculated solving a hydrostatic equation with the number density at the bottom being $7.3 \times 10^{12} \mathrm{~cm}^{-3}$. Our simulations are performed by solving the thermodynamic MHD equations given by

$$
\begin{aligned}
\frac{\partial \rho}{\partial t}+\nabla \cdot(\rho \boldsymbol{v}) & =0 \\
\frac{\partial(\rho \boldsymbol{v})}{\partial t}+\nabla \cdot\left(\rho \boldsymbol{v} \boldsymbol{v}+p_{\mathrm{tot}} \boldsymbol{I}-\frac{\boldsymbol{B} \boldsymbol{B}}{\mu_{0}}\right) & =\rho \boldsymbol{g} \\
\frac{\partial E}{\partial t}+\nabla \cdot\left(E \boldsymbol{v}+p_{\mathrm{tot}} \boldsymbol{v}-\frac{\boldsymbol{B} \boldsymbol{B}}{\mu_{0}} \cdot \boldsymbol{v}\right) & =\rho \boldsymbol{g} \cdot \boldsymbol{v}+H-R+\nabla \cdot(\boldsymbol{\kappa} \cdot \nabla T)
\end{aligned}
$$

$$
\frac{\partial \boldsymbol{B}}{\partial t}+\nabla \cdot(\boldsymbol{v} \boldsymbol{B}-\boldsymbol{B} \boldsymbol{v})=0
$$

where $\rho, \boldsymbol{v}, \boldsymbol{B}$, and $\boldsymbol{I}$ are the plasma density, velocity, magnetic field, and unit tensor, respectively. Moreover, $p_{\text {tot }} \equiv p+$ $B^{2} / 2 \mu_{0}$ is total pressure with gas pressure $p=2.3 n_{\mathrm{H}} k_{\mathrm{B}} T$ assuming full ionization and an approximate helium abundance $\left(n_{\mathrm{He}} / n_{\mathrm{H}}=0.1\right), E=p /(\gamma-1)+\rho v^{2} / 2+B^{2} / 2 \mu_{0}$ is total energy, and $\boldsymbol{g}=-g_{\odot} r_{\odot}^{2} /\left(r_{\odot}+z\right)^{2} \hat{z}$ is the gravitational acceleration with solar radius $r_{\odot}$ and the solar surface gravitational acceleration $g_{\odot}$. We use normalization units of length $L_{0}=10 \mathrm{Mm}$, time $t_{0}=1.43 \mathrm{~min}$, temperature $T_{0}=10^{6} \mathrm{~K}$, number density $n_{0}=10^{9} \mathrm{~cm}^{-3}$, velocity $v_{0}=116.45 \mathrm{~km} \mathrm{~s}^{-1}$, and magnetic field $B_{0}=2 \mathrm{G}$ to normalize the equations. Any number without a specified unit has the according normalization unit thoughout the paper. We use the Adaptive Mesh Refinement Versatile Advection Code (MPI-AMRVAC) (Keppens et al. 2012; Porth et al. 2014) to numerically solve these equations with a scheme setup combining the Harten-Lax-van Leer Riemann solver (Harten et al. 1983) with a third-order slope limited reconstruction (Čada \& Torrilhon 2009) and a three-step RungeKutta time integration. We add a diffusive term into the induction 
equation to keep the divergence of magnetic field under control (Keppens et al. 2003; van der Holst \& Keppens 2007; Xia et al. 2014). Thermal source terms in the energy equation, such as field-aligned $\left(\boldsymbol{\kappa}=\kappa_{\|} \boldsymbol{b} \boldsymbol{b}\right)$ thermal conduction and optically thin radiative cooling $R=1.2 n_{\mathrm{H}}^{2} \Lambda(T)$, are treated as in our previous studies (Xia et al. 2014; Xia \& Keppens 2016a) using a Super Time Stepping scheme (Meyer et al. 2012) and an exact integration scheme (Townsend 2009), respectively. To establish a hot corona, the global coronal heating is simulated by adding a parametrized heating term $H_{1}=c_{1} B^{1.75} n_{\mathrm{e}}^{0.125} / r^{0.75} \mathrm{erg} \mathrm{cm}^{-3} \mathrm{~s}^{-1}$ (Lionello et al. 2013; Mok et al. 2016), where $c_{1}=4.54$ is a constant, $B$ is magnetic field strength, $n_{\mathrm{e}}=1.2 n_{\mathrm{H}}$ is number density of electrons, and $r=1 /|(\nabla \boldsymbol{b}) \cdot \boldsymbol{b}|$ is the local radius of curvature of magnetic field lines, where $\boldsymbol{b}=\boldsymbol{B} / \boldsymbol{B}$ is a unit vector along the magnetic field. We split off the potential magnetic field as a time-invariant component, and numerically solve for the deviation from it (Tanaka 1994; Porth et al. 2014) to avoid negative pressure problems caused by errors in magnetic energy when magnetic energy dominates the internal energy in extremely low- $\beta$ plasma. On the four side boundaries, we use a symmetry boundary condition for density and gas pressure, and extrapolate magnetic field according to a zero-gradient assumption. On the bottom boundary, we fix the density, pressure, and magnetic field the same as in the initial condition. On the top boundary, we solve the hydrostatic equation to obtain density and pressure values assuming a continuous temperature distribution, and adopt the same treatment for magnetic field as on side boundaries. We use anti-parallel symmetry conditions for velocity to ensure zero velocity on the all boundary surfaces. The normal component of extrapolated magnetic field is modified to numerically fulfill a centered difference zero-divergence constraint.

The initial setup is not in an equilibrium, so we first run the simulation with only global heating in a relaxation period of $114 \mathrm{~min}$ to reach a quasi-equilibrium. Starting from that state, we reset the time to zero and add a localized heating to evaporate chromospheric plasma into the coronal loop to cool down to coronal rain. The localized heating $\mathrm{H}_{2}$ is concentrated at both foot point chromospheric regions of the arcade with strong vertical magnetic field and decays with height above $z=4 \mathrm{Mm}$ in a Gaussian profile:

$$
H_{2}= \begin{cases}f(t) c_{2}\left(B_{z} / B_{\mathrm{h}}\right)^{2} \mathrm{e}^{-\left(\left(z-z_{h}\right) / H_{\mathrm{m}}\right)^{2}} & \text { if } z>z_{\mathrm{h}} \\ f(t) c_{2}\left(B_{z} / B_{\mathrm{h}}\right)^{2} & \text { if } z \leq z_{\mathrm{h}},\end{cases}
$$

where $c_{2}=10^{-2} \mathrm{erg} \mathrm{cm}^{-3} \mathrm{~s}^{-1}, B_{\mathrm{h}}=68 \mathrm{G}, z_{\mathrm{h}}=4 \mathrm{Mm}, H_{\mathrm{m}}=$ $3.16 \mathrm{Mm}$, and $f(t)$ is a linear ramp function of time to switch on the heating smoothly and keep it fixed afterwards. This heating mimics long-term mild heating in nonflaring coronal loops, and can be modified to represent impulsive violent heating of flares to stimulate postflare loops and flare-driven coronal rain in the future. This final stage is simulated for $114 \mathrm{~min}$, and we present the results in the following sections.

\section{Results}

The localized heating evaporates chromospheric plasma into hot coronal plasma and causes upflows from footpoint regions with speeds peaking at about $50 \mathrm{~km} \mathrm{~s}^{-1}$ in the lower corona. A loop top region centered at $(0,0,40) \mathrm{Mm}$ takes the lead in cooling because the radiative cooling there dominates over heating and thermal conduction as a result of density enhancement. This enhancement is caused by the evaporated flows along the heating loops and by squeezing due to the expansion of underlying heated loops. At $13.5 \mathrm{~min}$, the central region cools down to $0.02 \mathrm{MK}$, below which temperature our radiative cooling vanishes. Then, induced siphon flows, with speeds up to $24 \mathrm{~km} \mathrm{~s}^{-1}$ coming from two sides, merge there and create a high-density low-temperature plasma blob. This blob grows in three dimensions and the most rapid extension happens along the direction parallel to the polarity inversion line ( $x$-axis) as shown in Fig. 1. This is caused by the similarity and concurrency of the condensation process across the loops with a similar shape and height distributed along the $x$-direction. Observations also found that condensations in neighboring magnetic field strands occur simultaneously, which was thought to be due to a common footpoint heating process (Antolin \& Rouppe van der Voort 2012). The head-on impact of siphon flows not only compresses the central cool plasma to form a dense plasma blob, but also at the same time launches two rebounding slow shocks propagating against the continuous siphon flows along the magnetic field. The shock fronts are clearly seen in the temperature and density maps on the slices through the condensation as shown in Figs. 1b-d. The shock-front surfaces are curved, slow shock fronts and show up on a bundle of similar magnetic flux tubes where condensations occur successively. The processes of condensation and shock formation on these flux tubes are essentially the same, although they start at different times. Therefore, the whole history of the condensation process in a single flux tube is represented by snapshots like Figs. 1b-d. Similar rebound shocks were also found in our previous $2.5 \mathrm{D}$ simulations (Xia et al. 2012; Fang et al. 2015). At this moment, the shocks decrease the inflow speed from about $50 \mathrm{~km} \mathrm{~s}^{-1}$ to about $17 \mathrm{~km} \mathrm{~s}^{-1}$, compress the plasma from $5 \times 10^{8}$ to $9 \times 10^{8} \mathrm{~cm}^{-3}$, and heat the plasma from $0.18 \mathrm{MK}$ to $0.21 \mathrm{MK}$.

As the condensation extends with a span of $37 \mathrm{Mm}$ across loop tops, it starts to distort and becomes fragmented in about 6 min due to RTI, which is shown in Fig. 2 where the vertical velocity pattern alternates upflows with downflows in a direction perpendicular to the magnetic field. The dense coronal rain plasma is gathered in falling spikes and upflows correspond to hot coronal plasma. The plasma $\beta$ of the condensation is about 0.5 and the magnetic field strength is about $2 \mathrm{G}$. The Atwood number $\boldsymbol{A}$, which is $\left(\rho_{\text {high }}-\rho_{\text {low }}\right) /\left(\rho_{\text {high }}+\rho_{\text {low }}\right)$, indicating the density disparity between two layers of fluid under acceleration in classical RTI theory (Chandrasekhar 1961), for the coronal rain plasma and underlying coronal plasma is about 0.93 . The coronal rain plasma is about 25 times denser than the underlying coronal plasma. Similar results were found in our previous studies (Moschou et al. 2015). As shown in Fig. 2c, the falling spikes of coronal rain plasma have larger growth rates and penetrate deeper into the opposite region than rising bubbles of coronal plasma as expected for Atwood numbers close to 1 . We note that the RTI modes in this study are almost perpendicular to local magnetic field, which should locally behave as in hydrodynamics where shortest wavelengths grow the fastest. However, since the magnetic field lines are line-tied at the bottom, the interchange deformation of magnetic field lines and the RTI mode growth is restrained.

The fragmented blobs move downward in three groups, namely, two large clumps at the two flanks where coronal loops are inclined, and one near the central vertical loops, as shown in the first row of Fig. 3. These blobs initially fall roughly in the vertical plane $(y=0)$ along the PIL. Because the evolution of our bipolar system is roughly symmetric and magnetic field is weak enough at those loop top regions, the blobs, sitting at loop tops with nearly zero projected gravity along the loops, can displace magnetic field transversally dragging down their hosting magnetic loops. Blobs of the central group fall slower than those at 

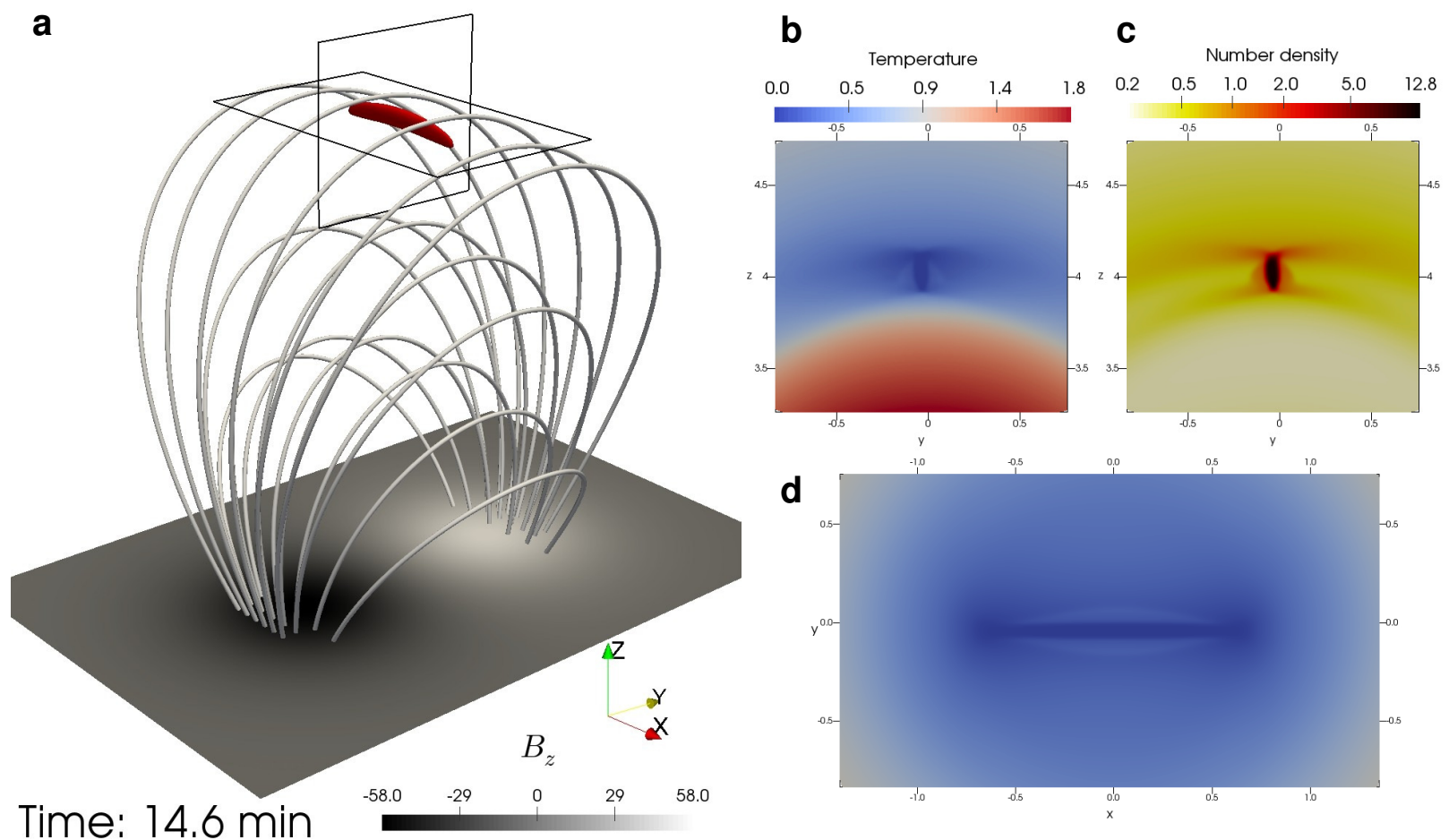

Fig. 1. a) A 3D view of the first condensed blob at 14.6 min shown by a red density isosurface at $7 \times 10^{9} \mathrm{~cm}^{-3}$ showing the condensed blob, magnetic field lines showing bipolar coronal loops, and bottom plane colored by vertical magnetic field. The field lines are integrated from fixed points at the bottom plane; a vertical slice in the $y-z$ plane $(x=0$, bounded by the vertical black frame in a)) through the condensation showing number density in panel b) and temperature in panel c); d) a horizontal slice in the $x-y$ plane $(z=40 \mathrm{Mm}$, bounded by the horizontal black frame in a) ) showing temperature structures with the same color-scale as in panel b) (numbers are dimensionless with according units of time $t_{0}$, magnetic field $B_{0}$, temperature $T_{0}$, and number density $n_{0}$ ). We note that all red isosurfaces and bottom magnetograms in the following figures have the same values as defined here.
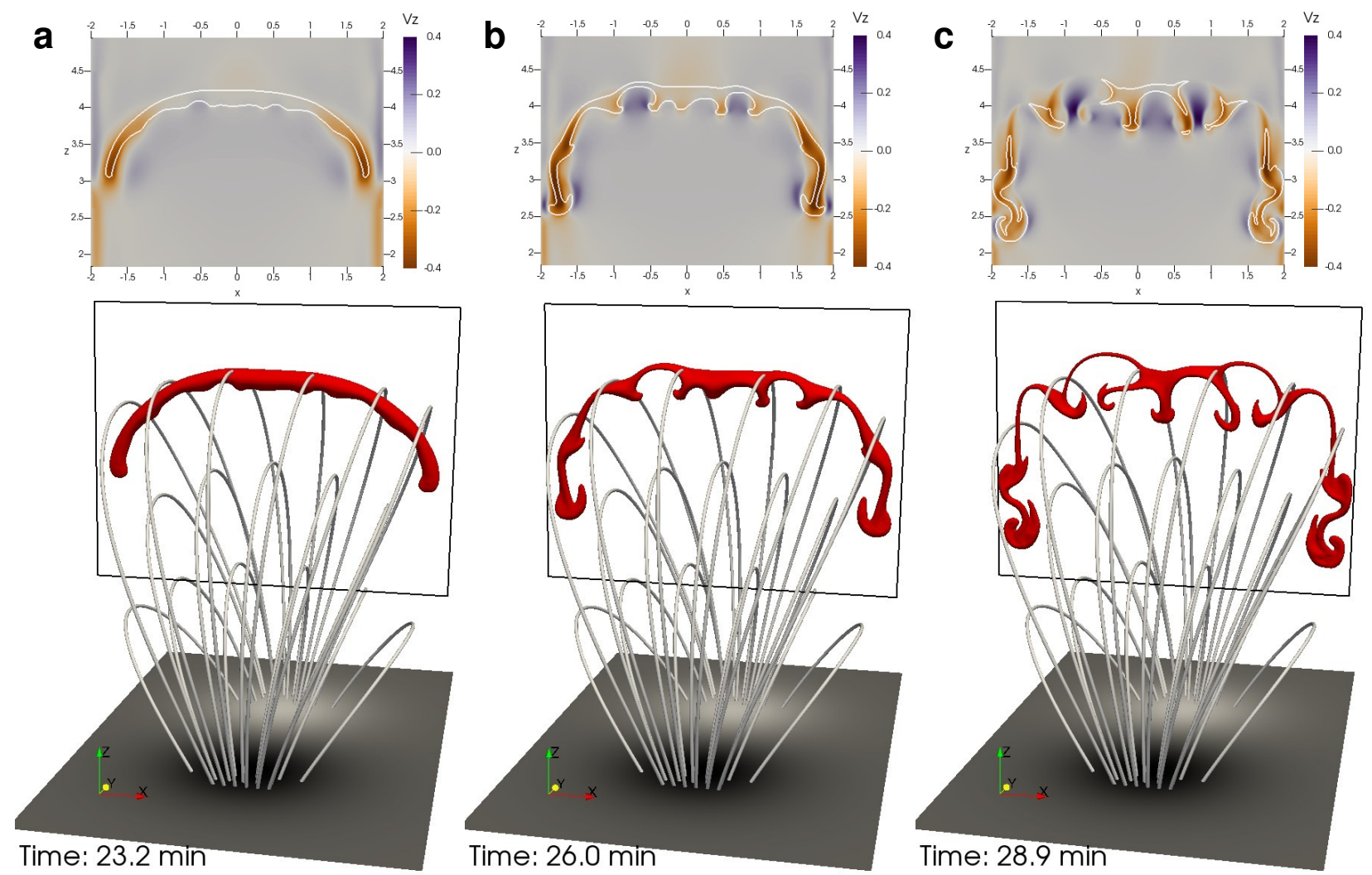

Fig. 2. Time series of snapshots (a) at $23.2 \mathrm{~min}$, b) at $26.0 \mathrm{~min}$, c) at $28.9 \mathrm{~min}$ ) presenting the fragmentation of the large condensation due to RTI. In the top row, vertical slices ( $x$ - $z$ planes), depicted by the black frames in the lower 3D views, are colored by the vertical component of velocity with white density contours at number density of $7 \times 10^{10} \mathrm{~cm}^{-3}$. 


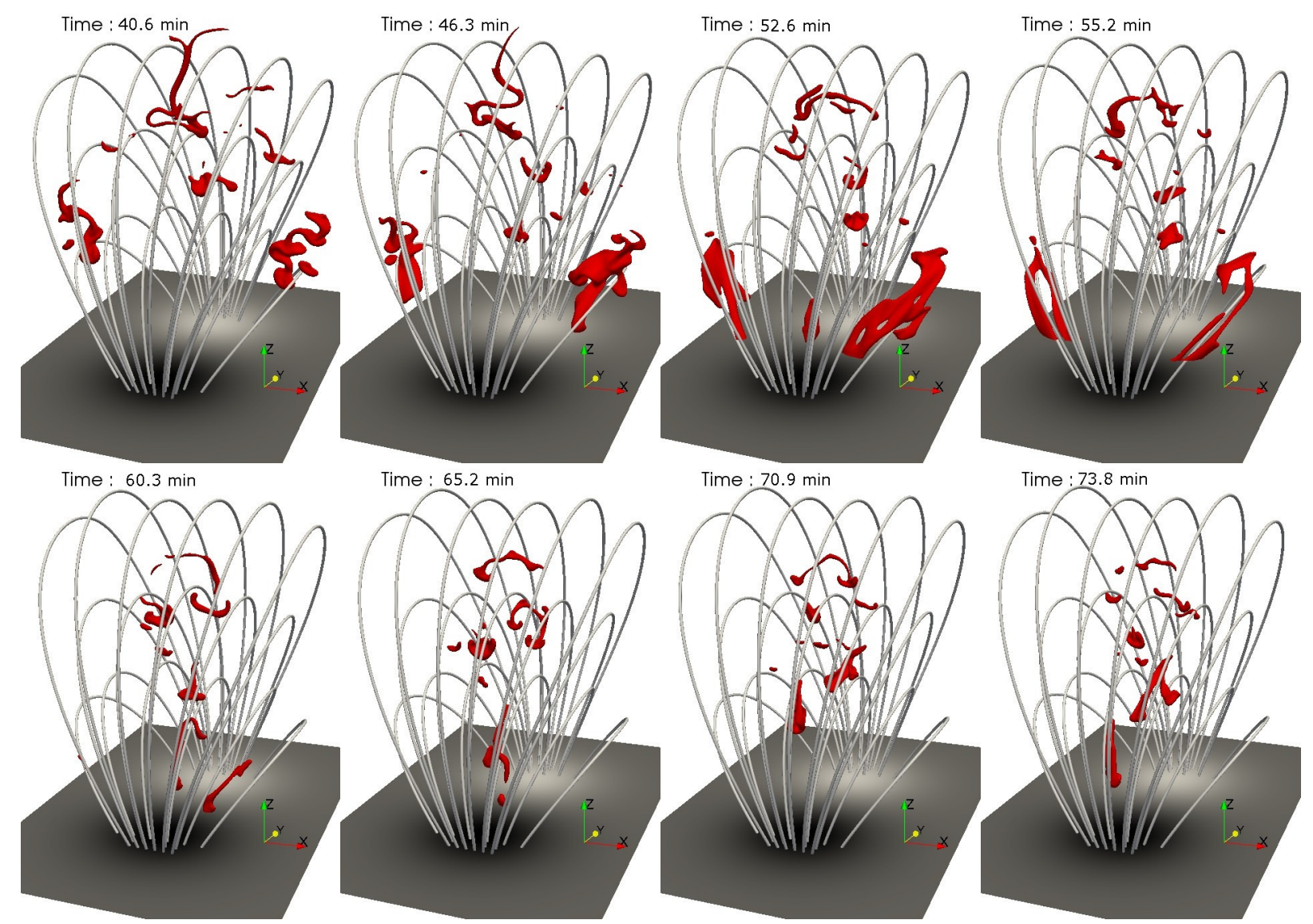

Fig. 3. Time series of snapshots on the evolution of coronal rain. Red density isosurfaces above a height of 4 Mm are plotted to show coronal rain blobs above the TR. Online Movie 1 shows the temporal evolution of these snapshots in a different viewing angle.

flanks due to the stronger resistant magnetic-tension force they encounter. They fragment further into smaller ones. Many tiny blobs gradually evaporate to hot plasma and disappear in the density isosurface view. The flank clumps fall almost vertically with speeds about $16 \mathrm{~km} \mathrm{~s}^{-1}$ until they reach a lower height of about $13 \mathrm{Mm}$. Then, they start to be more constrained in their motions by the local magnetic field with plasma $\beta$ about 0.1 and to be forced to stream down along the magnetic loops toward the foot points in the negative polarity region. After the flank blobs drain down completely, coronal rain blobs mainly form at the top region of the central loops and fall along the loops intermittently (see the second row of Fig. 3). During their fall, the condensed blobs keep changing shape, elongating, separating, or increasing in size.

In order to understand the time evolution, we quantify several properties of the coronal rain blobs in terms of mass, mass drainage rate, mean plasma $\beta$, angle between local velocity and magnetic field vector, instantaneous mean speed and maximal speed, and plot the time evolution curves in Fig. 4. Coronal rain blobs are cool and dense plasma above transition region, so we practically define that they are composed of cells with density larger than $7 \times 10^{10} \mathrm{~cm}^{-3}$, with temperature lower than $0.1 \mathrm{MK}$, and with height larger than $6 \mathrm{Mm}$. The mass drainage rate is the integrated coronal rain mass flux through a horizontal plane at $6.5 \mathrm{Mm}$ height. During the first condensation from $14 \mathrm{~min}$ to $30 \mathrm{~min}$, the condensation has a roughly linear growth rate of $2.84 \times 10^{9} \mathrm{~g} \mathrm{~s}^{-1}$, which is much slower than the one in $3 \mathrm{D}$ prominence simulation $\left(1.14 \times 10^{10} \mathrm{~g} \mathrm{~s}^{-1}\right)$ (Xia \& Keppens 2016a) and the estimation from prominence observations $\left(1.2 \times 10^{10} \mathrm{~g} \mathrm{~s}^{-1}\right)$ (Liu et al. 2012). Comparing with our previous $2 \mathrm{D}$ coronal rain simulations Fang et al. (2015, assuming $1000 \mathrm{~km}$ integral depth in the third direction), the condensation rate in this 3D model is an order of magnitude faster. From $30 \mathrm{~min}$ to $38 \mathrm{~min}$, the growth rate slows down because the fully developed RTI breaks the initial large blobs into pieces in which tiny blobs and very thin threads are heated to hotter and less dense material than coronal rain plasma mainly by thermal conduction. From $38 \mathrm{~min}$ until the first peak of the mass curve at $47.8 \mathrm{~min}$, the growth rate is about $3.25 \times 10^{9} \mathrm{~g} \mathrm{~s}^{-1}$, slightly faster than in the initial phase. After the first peak, the mass begins to drop rapidly when fast drainage due to falling of coronal rain starts. This fast drainage, which finishes at $60 \mathrm{~min}$, is contributed by the large falling clumps at the two flanks. After that, smaller coronal rain blobs formed at the central loop parts stream down episodically. As continuous condensation increases the coronal rain mass, the episodic fallen blobs, which correspond to the peaks in the mass drainage curve, decrease the mass. Therefore, the total mass remains at a steady level for a while from $55 \mathrm{~min}$ to $85 \mathrm{~min}$. After that, the mass increases and decreases forming a large hill in the curve over a period of $25 \mathrm{~min}$ when a rain shower forms and falls. The plasma $\beta$ of the condensation is relatively high (more than 0.5) at the beginning as the initial condensation forms in a weak magnetic field region at the loop top. With the solar gravity, RTI is inevitable under these circumstances and the nearly vertical falling of the condensation spikes pushes the magnetic loops down, making the angle between velocity and magnetic field close to 80 degrees. As the coronal rain blobs fall to lower regions with stronger magnetic field and smaller plasma $\beta$, they are ultimately forced to follow the guide of magnetic field lines with the angle decreasing. Therefore, the curve of plasma $\beta$ and 

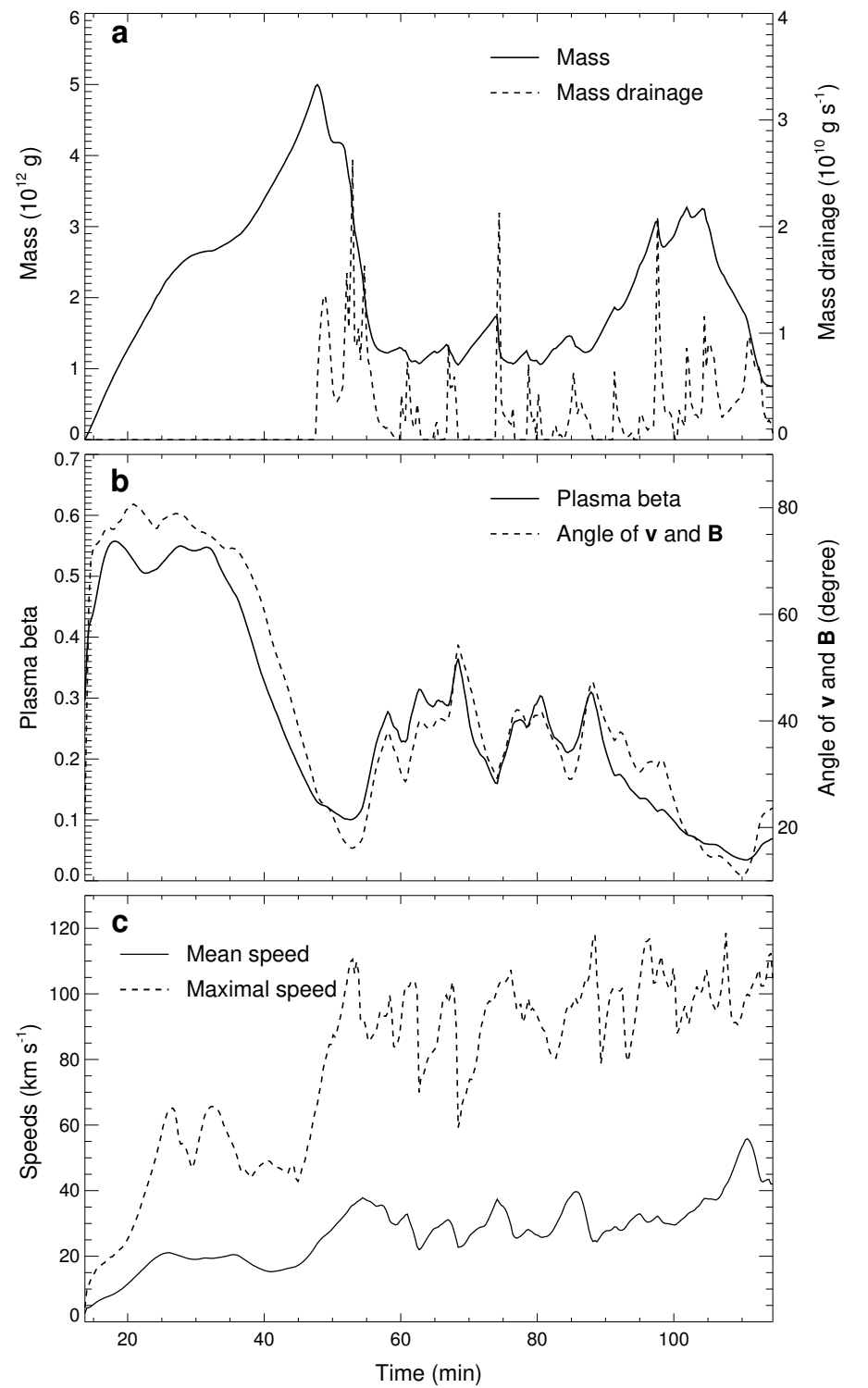

Fig. 4. Time evolution of coronal rain plasma properties shown as time curves of a) total coronal rain mass (solid line) and mass drainage rate (dashed line); b) mean plasma $\beta$ (solid line) and the angle between local velocity and magnetic field vector (dashed line); and c) instantaneous mean speed (solid line) and maximal speed (dashed line) of coronal rain blobs.

the angle match each other as shown in Fig. 4b. The mean speed of coronal rain plasma at each instant shifts from about $20 \mathrm{~km} \mathrm{~s}^{-1}$ to about $30 \mathrm{~km} \mathrm{~s}^{-1}$ after the fall of the large flank blobs, while the instantaneous maximal speed, which is about two to three times larger than the instantaneous mean speed, also increases to about $100 \mathrm{~km} \mathrm{~s}^{-1}$.

We wrote a program to automatically count the coronal rain blobs in each snapshot and quantify their properties, such as number, mass, volume, density, centroid location, and centroid speed (see Moschou et al. 2015, for details). The key algorithm is to group adjacent cells that contain coronal rain plasma into one blob, above TR, which is detected based on the vertical gradient of local density and temperature. To consider fully resolved blobs, we neglect tiny blobs containing less than 64 cells at the highest refinement level. Using this program, we performed statistical analysis of coronal rain blobs as shown in Fig. 5. The total number of blobs in all 352 snapshots is 2406 . The mean number
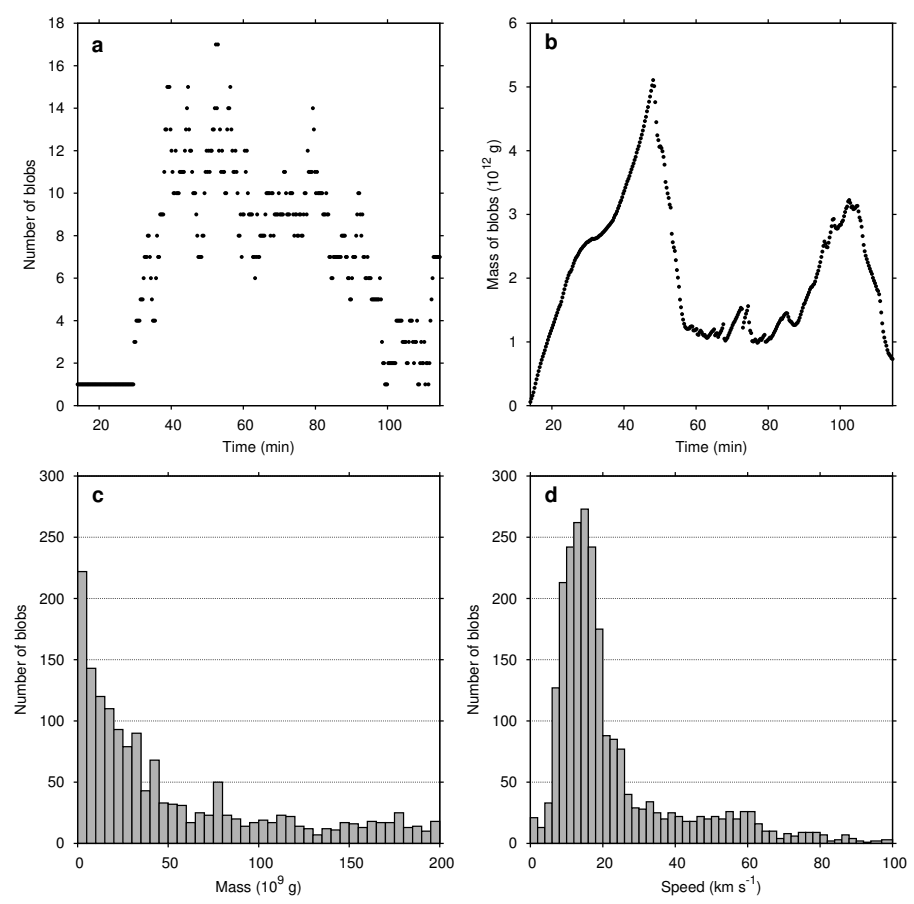

Fig. 5. Statistic analysis of coronal rain blobs. a) The number of coronal rain blobs at each instant; b) total mass of coronal rain blobs; c) distribution function of mass of blobs in all snapshots; and d) distribution function of speed of blobs in all snapshots.

of blobs per snapshot is 6.8. The maximal number of blobs in one snapshot is 17 . In the curve of blob number, we can see that the initial big blob breaks into three pieces at $30 \mathrm{~min}$. Then the number increases up to 17 at $55 \mathrm{~min}$ and decreases in the later phase. The total mass of coronal rain blobs matches well with the mass curve in Fig. 4a. Blobs are dominated by small ones with mass less than $5 \times 10^{10} \mathrm{~g}$ with the heaviest blob of $3.2 \times 10^{12} \mathrm{~g}$. The distribution of speed has a peak around $15 \mathrm{~km} \mathrm{~s}^{-1}$ and a long tail over $100 \mathrm{~km} \mathrm{~s}^{-1}$. The mean speed of all blobs is $22.4 \mathrm{~km} \mathrm{~s}^{-1}$. The blob with the maximal speed of $104.5 \mathrm{~km} \mathrm{~s}^{-1}$ is very small with a mass of $2.4 \times 10^{9} \mathrm{~g}$.

After analyzing the overall properties of the modeled coronal rain event, we go into more detail with case studies of typical coronal rain blobs. In Fig. 6, we find several coronal rain blobs, presented by red density isosurfaces, at different heights in different time stages of their life. We focus on a stretched coronal rain blob in the lower left part of panel (a). It is falling roughly in the central plane with $x$ close to 0 . It has a swelled head with relatively high speed $\left(50 \mathrm{~km} \mathrm{~s}^{-1}\right)$ before hitting the TR, a straight elongated body, and a hook-like tail. The blob is falling along a bundle of magnetic loops with its lower parts residing on the shorter loops, as shown by two field lines going through the head and the tail part. A vertical slice cutting through the blobs shows the $v_{y}$ distribution. There are fast following flows in the wake of the falling blob. The elongating blob has low gas pressure in its body inducing siphon flows that enhance the wake flows (see the blue regions). The siphon flows upstream of the falling blob become neutralized by the pushing motion of the blob. Blobs near the loop top in higher regions have little motion along the loops, so the siphon flows from both sides towards the blobs are obvious and bring material to feed the growth of the blobs. Once the blobs reach the low- $\beta$ region, the lower part at the lower loops feels stronger projected gravity due to the shape of the loops. The lower part falls faster than the upper part along the 

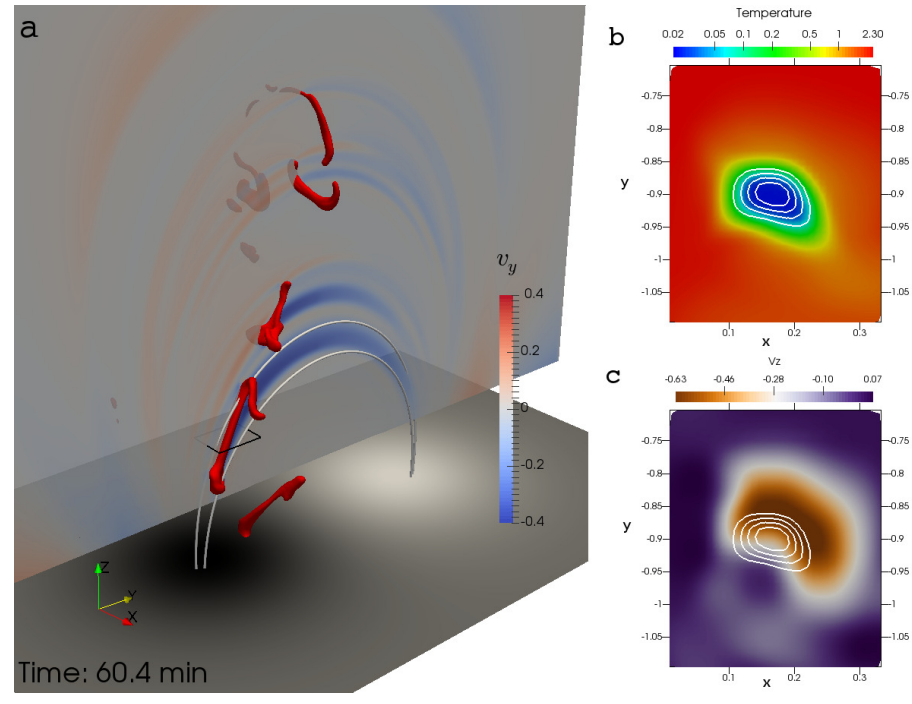

Fig. 6. Case study of a stretched coronal rain blob at $60.4 \mathrm{~min}$; a) shows a $3 \mathrm{D}$ view of blobs in red isosurface of density, two magnetic field lines going through the head and tail of a blob, a vertical plane cutting through the blobs with translucent color indicating $v_{y}$; b) shows the horizontal slice through the blob bounded by the black frame in a) colored by temperature with number density contours in values of 7,11 , $15,19,23 n_{0}$; and c) shows the slice colored by $v_{z}$ with the same density contours as b).

loops, so an initially compact blob gets elongated and this tearing also decreases the gas pressure in the straight body of an elongated blob. Besides, when a plasma blob streams down in a flux tube with narrower cross-section closer to the footpoints, it will be squeezed and elongated. We made a small horizontal slice cutting across the selected blob, and plot the temperature map (panel (b)) and $v_{z}$ map (panel (c)) with number density contours on them. The blob is wrapped by a TR which connects the $0.04 \mathrm{MK}$ coronal rain plasma to $1 \mathrm{MK}$ coronal plasma in a range of about $500 \mathrm{~km}$. The thickness of the blob in this crosssection is about $700 \mathrm{~km}$ if we count the $7 n_{0}$ density contour and $0.1 \mathrm{MK}$ temperature contour as its border. The density contours and the temperature map show increasing density and decreasing temperature when getting closer to the center of the blob, which reflects the multi-density and multi-thermal nature of the coronal rain blobs (Antolin et al. 2015). In the vertical velocity map, the downflow region occupies not only the coronal periphery but also half the blob itself. These anti-parallel shearing flows are divided over the core of the blob, which leads to elongation of the blob during its fall. The large velocity difference between different parts of individual blobs is also reflected in the significant difference between the maximal speed and the mean speed at each snapshot in Fig. 4c.

To directly compare with observed images from the Solar Dynamics Observatory Atmospheric Imaging Assembly (AIA) instrument, we made synthetic observations on our simulations in selected viewing angle and retrieved synthetic images with a technique described in detail in Xia et al. (2014). To mimic absorption of EUV emission by the core plasma of condensations, we exclude emission coming from behind dense plasma with a number density larger than $2 \times 10^{10} \mathrm{~cm}^{-3}$, except for the $304 \AA$ wave channel. For example, Fig. 7 shows synthetic views on the modeled coronal rain and coronal loops at 60.4 min in $211 \AA$, $193 \AA, 171 \AA$, and $304 \AA$ wave channels, which have main contribution temperatures of about $1.8,1.5,0.8$, and $0.08 \mathrm{MK}$, respectively. The line of sight of Fig. 7 has an azimuthal angle
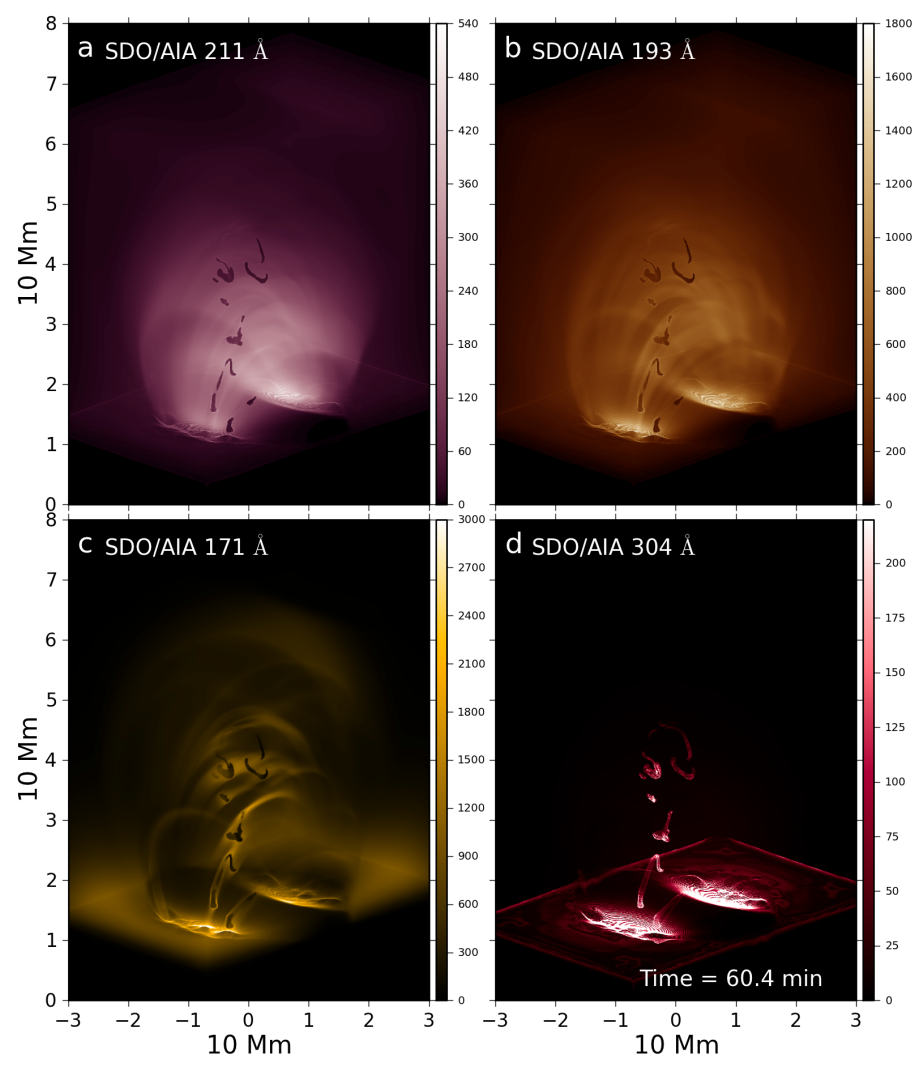

Fig. 7. SDO/AIA synthetic views on coronal rain blobs at $60.4 \mathrm{~min}$ in a) $211 \AA$; b) $193 \AA$; c) $171 \AA$; and d) $304 \AA$ wave channels. Numbers in the color bars have a unit of $\mathrm{DN} \mathrm{s}^{-1}$. Movie 2 showing this figure is available online.

of $45^{\circ}$ away from the PIL and an elevation angle of $20^{\circ}$. In hot channels panel (a), (b), and (c), the bright coronal loops and their footpoint regions appear more dispersed in higher-temperature channels. Dark clumps of coronal rain reside in dim loops in $211 \AA$, while adhering to bright halo segments of $171 \AA$ loops. Bright clumps of coronal rain are found in the cooler $304 \AA$ channel sampling TR plasma. The impact site of coronal rain at the chromosphere is at this moment a fairly compact region of negative polarity with a diameter less than $10 \mathrm{Mm}$. In the movie of Fig. 7, the inital condensation is barely seen in $304 \AA$, because the density of the emission-contributing plasma (around $0.08 \mathrm{MK}$ ) is relatively low (about $2 \times 10^{9} \mathrm{~cm}^{-3}$ ) at the transition layer between the condensation and corona.

In the last phase of the simulation from $90 \mathrm{~min}$ to $113 \mathrm{~min}$, a bursty condensation makes a coronal rain "shower" in a large bundle of coronal loops. The total mass of the condensation increases and peaks at $105 \mathrm{~min}$. The total number of blobs first increases and soon decreases because neighboring small condensations grow fast enough to touch one another and to be counted as one blob. We plot a representative snapshot of this phase in Fig. 8. As shown by density isosurfaces in panel (a), long streams of condensed plasma are connected to one another. The horizontal extension of the streams along the $x$-direction, covering loops with similar length, is about $10 \mathrm{Mm}$, while the thickness of the streams along $y$-direction is around $1 \mathrm{Mm}$. In panel (b), a horizontal cross-section of the streams finds the density inhomogeneities within the coronal rain clumps, which is similar to the results found in observations (Antolin et al. 2015), although the spatial sizes are different. In our model, a coronal rain clump can have density inhomogeneity because it is composed 

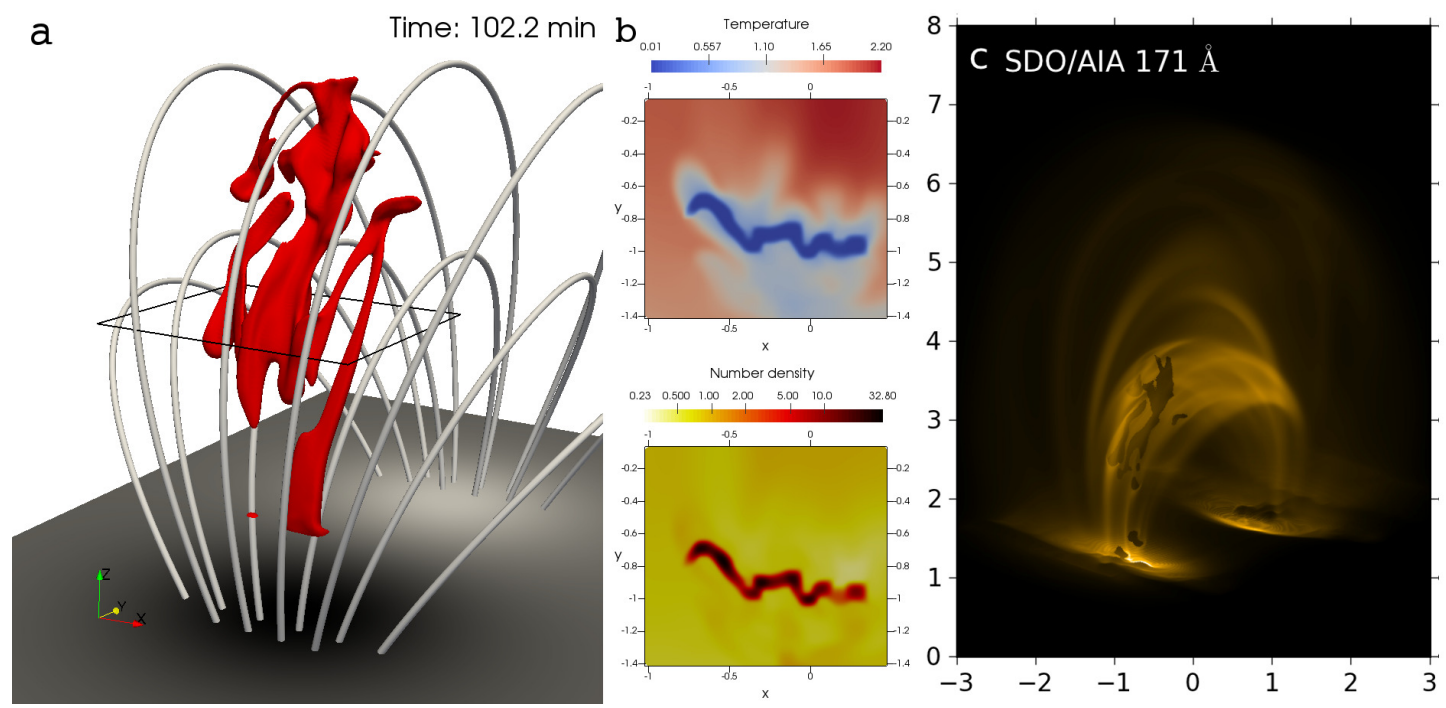

Fig. 8. Coronal rain "shower" at $102.2 \mathrm{~min}$; a) shows a 3D view with red density isosurfaces and selected magnetic field lines; b) shows a plane slice across the rain blobs, indicated by the black frame in a), howing temperature map and number density map; and c) shows a synthetic view in AIA $171 \AA$ wave channel.

of several seed blobs which grow and move along the magnetic loops closely in space and time. In panel (c), a synthetic view in AIA $171 \AA$ channel shows dark clumps, which are the highdensity $\left(2 \times 10^{10} \mathrm{~cm}^{-3}\right)$ ores of coronal rain streams in panel (a), in bright coronal loops.

\section{Conclusions and discussion}

We realized a 3D coronal rain simulation with unprecedented high resolution and a more realistic magnetic environment than previous models. The formation process of coronal rain, characterized by progressive cooling and condensation in situ, is reproduced in our model. The physical reason behind it is the radiation-dominated thermal instability. The formation of the first condensation is presented in detail with its 3D shape extending along the PIL and with the shape of rebounding shock fronts indicating the time sequence of the condensation progress. The first long condensation on the loop top region develops RTI interchange deformation and becomes fragmented into smaller blobs. The blobs fall slowly and vertically against magnetic field loops until reaching low- $\beta$ regions and start to slip and fall along the field loops from loop top to loop footpoints. A similar phenomenon was presented by Petralia et al. (2016) in a 3D MHD simulation of the falling of dense fragments along magnetic flux tubes of an erupted solar filament, in which the initial falling speed of the dense blobs is about $300 \mathrm{~km} \mathrm{~s}^{-1}$, much higher than our case, and fast enough to generate slow shocks, which are not found ahead of the falling blobs in our study. They also found that these fast-moving blobs with imposed initial motions misaligned with magnetic field soon become deformed and mixed due to the feedback from dragged magnetic field lines (Petralia et al. 2017). Similarly, the misaligned motion of our falling blobs near the loop tops also leads to reshaping and fragmentation of the blobs.

Statistical study of the coronal rain blobs found in all snapshots in our simulation shows that small blobs with mass less than $10^{10} \mathrm{~g}$ dominate the population despite the fact that unusually large blobs show up in the initial and last phases. Large blobs have statistically slower centroid speed than small ones. When the blobs stream along the magnetic loops, they are stretched and develop a non-uniform velocity pattern. The elongated blobs have faster (siphon) flow on those parts closer to the center of curvature of the hosting coronal loops. AIA synthetic observations of our simulated coronal rain well resemble real observations in several aspects, like dark falling clumps in hot EUV channels and bright rain blobs in cool $304 \AA$ channel. We also find density inhomogeneities within the coronal rain clumps in a rain "shower", which may explain the observed multi-stranded nature of coronal rain (Antolin et al. 2015).

The magnetic field strength in this model is as yet relatively low compared to typical active region coronal loops at the same height. It is computationally challenging to simulate plasma with extremely low $\beta$ and high Alfvén speed when solving conservative energy equations and using explicit schemes. At the cost of much more computational resources, we can get closer to the realistic magnetic field strength in the work in progress. The RTI interchange mode of loop-top condensations may then be suppressed by the rigid line-tied coronal loops in very low- $\beta$ regimes. The physics of rain-blob formation and the dynamics of coronal rain blobs in the lower regions should remain the same. Since the RTI interchange mode of prominence plasma has been observed (Berger et al. 2008, 2010) and modeled (Hillier et al. 2011; Keppens et al. 2015; Xia \& Keppens 2016b) in the quiescent region, it may be found on high- $\beta$ loop-top by future observations. Previous simulations of RTI in prominences start with existing static prominence material excluding the dynamic formation process; the model here is the first one covering both formation and subsequent RTI of prominence-like plasma in similar local magnetic environment, for example, weak and locally horizontal magnetic field. However, the vertical thickness of the initial condensation is only about $2 \mathrm{Mm}$, much smaller than a typical prominence and the bipolar magnetic structure is quite different from prominence-hosting magnetic structures with magnetic dips, thus the details of RTI here are different from those in modeled prominences and observed prominences. We further plan to use realistic magnetic field extrapolated from actual observed magnetograms and study the dynamics of coronal rain blobs. Flare-driven coronal rain has not yet been simulated and needs simulations for further understanding of its fine-scale strands and the cooling of post-flare loops. 
Acknowledgements. C.X. wishes to thank FWO (Research Foundation Flanders) for the award of postdoctoral fellowship. C.X. appreciates the suggestive discussion with Dr. Patrick Antolin. This research was supported by FWO and by KU Leuven Project No. GOA/2015-014 and by the Interuniversity Attraction Poles Programme by the Belgian Science Policy Office (IAP P7/08 CHARM). The simulations were conducted on the VSC (Flemish Supercomputer Center funded by Hercules foundation and Flemish government).

\section{References}

Antiochos, S. K., MacNeice, P. J., Spicer, D. S., \& Klimchuk, J. A. 1999, ApJ 512,985

Antolin, P., \& Rouppe van der Voort, L. 2012, ApJ, 745, 152

Antolin, P., \& Verwichte, E. 2011, ApJ, 736, 121

Antolin, P., Shibata, K., \& Vissers, G. 2010, ApJ, 716, 154

Antolin, P., Vissers, G., Pereira, T. M. D., Rouppe van der Voort, L., \& Scullion, E. 2015, ApJ, 806, 81

Aschwanden, M. J. 2001, ApJ, 560, 1035

Aschwanden, M. J., Schrijver, C. J., \& Alexander, D. 2001, ApJ, 550, 1036

Berger, T. E., Shine, R. A., Slater, G. L., et al. 2008, ApJ, 676, L89

Berger, T. E., Slater, G., Hurlburt, N., et al. 2010, ApJ, 716, 1288

Cada, M., \& Torrilhon, M. 2009, J. Comput. Phys., 228, 4118

Chandrasekhar, S. 1961, Hydrodynamic and hydromagnetic stability (Oxford Clarendon)

de Groof, A., Bastiaensen, C., Müller, D. A. N., Berghmans, D., \& Poedts, S. 2005, A\&A, 443, 319

De Pontieu, B., McIntosh, S., Hansteen, V. H., et al. 2007, PASJ, 59, S655

De Pontieu, B., McIntosh, S. W., Carlsson, M., et al. 2011, Science, 331, 55

Fang, X., Xia, C., \& Keppens, R. 2013, ApJ, 771, L29

Fang, X., Xia, C., Keppens, R., \& Van Doorsselaere, T. 2015, ApJ, 807, 142

Field, G. B. 1965, ApJ, 142, 531

Hansteen, V. H., Hara, H., De Pontieu, B., \& Carlsson, M. 2010, ApJ, 718, 1070

Harten, A., Lax, P. D., \& van Leer, B. 1983, SIAM Rev., 25, 35

Hillier, A., Isobe, H., Shibata, K., \& Berger, T. 2011, ApJ, 736, L1

Kamio, S., Peter, H., Curdt, W., \& Solanki, S. K. 2011, A\&A, 532, A96

Karpen, J. T., Antiochos, S. K., Hohensee, M., Klimchuk, J. A., \& MacNeice, P. J. 2001, ApJ, 553, L85
Kawaguchi, I. 1970, PASJ, 22, 405

Keppens, R., Nool, M., Tóth, G., \& Goedbloed, J. P. 2003, Comput. Phys. Commun., 153, 317

Keppens, R., Meliani, Z., van Marle, A. J., et al. 2012, J. Comput. Phys., 231, 718

Keppens, R., Xia, C., \& Porth, O. 2015, ApJ, 806, L13

Lionello, R., Winebarger, A. R., Mok, Y., Linker, J. A., \& Mikić, Z. 2013, ApJ, 773,134

Liu, W., Berger, T. E., \& Low, B. C. 2012, ApJ, 745, L21

Meyer, C. D., Balsara, D. S., \& Aslam, T. D. 2012, MNRAS, 422, 2102

Mok, Y., Mikić, Z., Lionello, R., Downs, C., \& Linker, J. A. 2016, ApJ, 817, 15 Moschou, S. P., Keppens, R., Xia, C., \& Fang, X. 2015, Adv. Space Res., 56, 2738

Müller, D. A. N., Hansteen, V. H., \& Peter, H. 2003, A\&A, 411, 605

Müller, D. A. N., Peter, H., \& Hansteen, V. H. 2004, A\&A, 424, 289

Müller, D. A. N., De Groof, A., Hansteen, V. H., \& Peter, H. 2005, A\&A, 436 1067

O’Shea, E., Banerjee, D., \& Doyle, J. G. 2007, A\&A, 475, L25

Parker, E. N. 1953, ApJ, 117, 431

Petralia, A., Reale, F., Orlando, S., \& Testa, P. 2016, ApJ, 832, 2

Petralia, A., Reale, F., \& Orlando, S. 2017, A\&A, 598, L8

Pettit, E. 1943, ApJ, 98, 6

Porth, O., Xia, C., Hendrix, T., Moschou, S. P., \& Keppens, R. 2014, ApJ, 214, 4

Rouppe van der Voort, L., Leenaarts, J., De Pontieu, B., Carlsson, M., \& Vissers, G. 2009, ApJ, 705, 272

Schrijver, C. J. 2001, Sol. Phys., 198, 325

Scullion, E., Rouppe van der Voort, L., Wedemeyer, S., \& Antolin, P. 2014, ApJ, 797, 36

Scullion, E., Rouppe van der Voort, L., Antolin, P., et al. 2016, ApJ, 833, 184

Tanaka, T. 1994, J. Comput. Phys., 111, 381

Török, T., \& Kliem, B. 2003, A\&A, 406, 1043

Townsend, R. H. D. 2009, ApJ, 181, 391

van der Holst, B., \& Keppens, R. 2007, J. Comput. Phys., 226, 925

Xia, C., \& Keppens, R. 2016a, ApJ, 823, 22

Xia, C., \& Keppens, R. 2016b, ApJ, 825, L29

Xia, C., Chen, P. F., Keppens, R., \& van Marle, A. J. 2011, ApJ, 737, 27

Xia, C., Chen, P. F., \& Keppens, R. 2012, ApJ, 748, L26

Xia, C., Keppens, R., Antolin, P., \& Porth, O. 2014, ApJ, 792, L38 